\title{
Curcumin Treatment Suppresses CCR7 Expression and the Differentiation and Migration of Human Circulating Fibrocytes
}

\author{
Xu-yan Fu $\quad$ Dong-wei Zhang ${ }^{b} \quad$ Ya-dong Lia ${ }^{a}$ Pi-wen Zhao ${ }^{a} \quad$ Yu-qing Tang ${ }^{a}$ \\ Jian-zhao Niu ${ }^{a}$ Yu Lia \\ aSchool of Preclinical Medicine, Beijing University of Chinese Medicine, biabetes Research Center, \\ Beijing University of Chinese Medicine, Beijing, China
}

\section{Key Words}

Human Circulating Fibrocytes $\cdot$ Curcumin $\cdot \mathrm{CCR} 7 / \mathrm{CCL} 21$ pathway $\bullet$ Migration $\cdot$ Activation

\begin{abstract}
Background/Aim: Recent studies have demonstrated that circulating fibrocytes contribute to the formation and development of fibrosis. Curcumin, a polyphenolic compound isolated from turmeric, has been shown to have anti-fibrotic effects in various organs. We and others have demonstrated that curcumin beneficially affects the development of fibrosis. However the effect of curcumin on circulating fibrocytes has not been reported. Methods: Human circulating fibrocytes were isolated from leukocyte concentrates of healthy human donors and identified based on the expression of CD34, CD45, collagen I (COLI), and chemokine receptor CCR7 (CCR7) via flow cytometry. Cell Counting Kit-8 was used to evaluate cell viability. The effect of curcumin on the differentiation and migration of human circulating fibrocytes was evaluated by immunofluorescence staining, flow cytometry and a transwell migration assay. Transforming growth factor (TGF)- $\beta 1$ secretion was examined by ELISA. Results: Curcumin treatment $(72 \mathrm{~h} ; 20 \mu \mathrm{M})$ significantly decreased the expression of COL I, $\alpha$-SMA and CCR7, as well as TGF- $\beta$ I secretion, in human circulating fibrocytes. The inhibitory effect of curcumin on the differentiation and migration of human circulating fibrocytes is likely via regulating the CCR7/CCL21 signaling pathway, in particular by reducing CCR7 expression. These observed effects may be beneficial in resolving fibrosis by suppressing TGF- $\beta 1$ secretion. Conclusion: Our results suggest that curcumin has the potential to suppress the differentiation and migration of circulating fibrocytes, which would provide new explanation for curcumin's application in the development of fibrosis in various organs.

X.-y. Fu and D.-w. Zhang contributed equally.

Yu Li, PhD, MD

KARGER 125 


\section{Introduction}

Fibrosis is a pathological status characterized by excessive and persistent accumulation of fibrous connective tissue in an organ in response to pathological factors such as injury and inflammation $[1,2]$. Scaring and the development of excess fibrous connective are two major symptoms of the fibrosis, and can obliterate the architecture and function of the underlying organ or tissue [3]. The symptoms of fibrosis may vary considerably depending on the location and extent of the condition as well as the symptoms of the underlying cause. This condition causes high morbidity and mortality globally [4], and limited therapeutic options are available for controlling the development of fibrosis thus far. It is widely acknowledged that (myo) fibroblasts are the main sources of extraceluar matrix (ECM) production [1]. Discovered by Bucala [5] in 1994, blood-borne circulating fibrocytes have been reported to be involved in pulmonary [6], renal $[7,8]$, liver $[7,9,10]$ and cardiac $[7,11]$ fibrosis. Derived from bone marrow, circulating fibrocytes exist in the peripheral circulation and migrate with the peripheral blood to wound chambers in response to chemokines [12]. Activated circulating fibrocytes not only secrete ECM, which is essential for the process of fibrosis, but also lead to pathological fibrosis by differentiating to (myo) fibroblasts [13]. This prompted us to investigate whether targeting circulating fibrocytes could open up a new avenue for resolving fibrosis.

Curcumin, a polyphenolic compound isolated from turmeric (Zingiberaceae), has been shown to exert a variety of biological effects such as anti-hypolipidemic, anti-hypoglycemic, anti-tumor, anti-oxidant and anti-inflammatory effects [14]. Increasing evidence suggests that curcumin has antifibrotic effects on cardiac fibrosis in spontaneously hypertensive rats via PPAR- $\gamma$ activation [15]; on liver fibrosis in carbon tetrachloride treated rats via inhibition of transforming growth factor 1 and connective tissue growth factor expression[16], suppressing cannabinoid receptor type-1 [17], as well as ameliorating intrahepatic angiogenesis [18]; on renal fibrosis in unilateral ureteral obstruction (UUO) rat via inhibiting of nuclear factor kappa-B and activator protein-1[19] and in 5/6 nephrectomized rat via modulating Nrf2Keap1 pathway [20]; on pulmonary fibrosis in viral-induced acute respiratory distress syndrome mice through the alteration of inflammation and myofibroblast differentiation [21] and in bleomycin treated mice via overexpression of cathepsins $\mathrm{K}$ and L [22].

Thus, we speculated that curcumin has anti-fibrotic effects in various organs by targeting circulating fibrocytes. In order to test the hypothesis, we established methods for the culture and identification of human circulating fibrocytes, and evaluated the effects of curcumin on their viability, differentiation, and migration, as well as the possible mechanism of these effects.

\section{Materials and Methods}

\section{Cell culture}

Human circulating fibrocytes were isolated from leukocyte concentrates from healthy human donors (Beijing Red Cross Blood Center, China; the experiment has been carried out in accordance with The Code of Ethics of the World Medical Association (Declaration of Helsinki) and approved by the Medical Ethics committee of the Beijing University of Chinese Medicine) by centrifugation over Ficoll-Plaque PLUS (GE Healthcare, USA) according to the manufacturer's protocol. The leukocyte concentrate was diluted with phosphate-buffered saline (PBS; Macgene, China) at a volume ratio of 1:1. Subsequently, this diluted blood sample was layered on Percoll-Paque PLUS in a centrifuge tube. After being centrifuged at $400 \times \mathrm{g}$ for 30 minutes at room temperature, the lymphocyte layer was carefully transferred into a clean centrifuge tube containing at least 3 volumes of PBS. Cells were washed twice at $100 \times \mathrm{g}$ for 10 minutes. Following culture on T25 plastic dishes at a density of $0.75 \times 10^{6}$ cells $/ \mathrm{cm}^{2}$ for 3 days in Dulbecco's Modified Eagle Medium (DMEM; Gibco, USA) with 20\% fetal bovine serum (Gibco), L-glutamine, penicillin and streptomycin (Thermo, USA), non-adherent cells were removed. New media were applied to the adherent cells and cells remained in culture for the following experiments.

\section{KARGER}


Immunofluorescence

Cells were seeded into $35 \mathrm{~mm}$ Petri dishes for 5 days and immunostained with phycoerythrin (PE)conjugated mouse anti-human CD34 monoclonal antibody (1/50; BD Biosciences, USA), PE-conjugated rat anti-human C-C chemokine receptor type 7 (CCR7) monoclonal antibody (1/50; BD Biosciences, USA) or PE-Cy5-conjugated mouse anti-human CD45 monoclonal antibody (1/50; BD Biosciences) or PEconjugated rat anti-human CCR7 monoclonal antibody (1/50; BD Biosciences) for 30 minutes at $4^{\circ} \mathrm{C}$. After cells were washed three times, they were fixed and permeabilized by the addition of BD cytofix/cytoperm (BD Biosciences) for 20 minutes at $4^{\circ} \mathrm{C}$. After washing and blocking, the cells were incubated for 30 minutes with mouse anti-human collagen I (COL I) primary antibody (1/200; Abcam). After the cells were washed three times with BD Perm/Wash ${ }^{\mathrm{TM}}$ buffer, fluorescein isothiocyanate (FITC)-labeled rabbit anti-mouse IgG H\&L secondary antibody (1/200; Abcam, UK) against rabbit anti-mouse collagen I was added and incubated for $30 \mathrm{~min}$, and the cells were then stained with 4,6-diamidinophenyl-2-indole (DAPI) (Roche, Germany), and viewed on a laser scanning microscope (OLYMPUS, Japan) using FV-ASW ver.3.1a software. Cells were identified by double immunocytochemistry with either CD34 and COL I, CD45 and COL I, CCR7 and COL I, or CD45 and CCR7.

\section{Flow cytometry analysis}

For flow cytometry analysis, $0.75 \times 10^{6}$ cells were collected and centrifuged. Cells were stained for CD34 (as described in the immunofluorescence section, same below), CD45, COL I, CCR7 or $\alpha$-smooth muscle actin ( $\alpha$-SMA, rabbit anti-human primary antibody, 1/100; Millipore, USA; allophycocyanin (APC)-labeled goat anti-rabbit IgG H\&L secondary antibody, 1/100; Southern Biotech, USA). After immunostaining, cells were washed twice and analyzed by flow cytometry (BD FACS Cantoll, USA) using BD FASDiva software and FlowJo 7.6.1 software. Negative controls used for non-specific background staining included: PElabeled mouse IgG1 isotype control (BD Biosciences, USA), PE-Cy5-labelled mouse IgG1 isotype control (BD Biosciences, USA), FITC-labeled purified mouse IgG1 $\kappa$ isotype control (BD Biosciences, USA), PE-labeled rat IgG2a $\kappa$ isotype control (BD Biosciences), APC-labeled rabbit IgG isotype control (Epitomics, USA).

\section{Cell viability assay}

Human circulating fibrocytes were seeded at $5 \times 10^{4}$ viable cells in a 96 well tissue culture plate in complete media (20\% FBS in DMEM) for 3 days. The media was removed and replaced with new complete media in the presence or absence of various concentrations of curcumin (Sigma, USA; 1, 3, 10 and $20 \mu \mathrm{M}$ ). Cells were cultured for 24, 48, or 72 hours before the addition of serum free media with Cell Counting Kit-8 (CCK-8; DOJINDO, Japan) for an additional 3 hours. The optical density (OD) at 450nm was recorded using a microplate reader.

\section{ELISA for transforming growth factor beta 1(TGF- $\beta 1$ )}

After the cells were cultured for 10 days, the media were removed and replaced with new complete media containing various concentrations of curcumin $(0,1,3,10$ and $20 \mu \mathrm{M})$. The blank control group was set. The experiment was conducted according to the guidelines of the ELISA kit (eBioscience, USA). In brief, following the exposure of cells to curcumin for 72 hours, the culture supernatants were collected and prediluted with the assay buffer. The pretreated samples were added into wells and incubated for 2 hours. Then, after the addition of biotin-conjugate for 1 hour, the samples were incubated with TMB substrate solution for 1 hour. The reaction was then halted by the addition of the stop solution and the plate was read in a plate reader at $450 \mathrm{~nm}$.

\section{Transwell migration assay}

Twenty-four-well chemotaxis chambers (Corning, USA) were used in the chemotaxis assays. The chambers were balanced in serum-free medium for 1 hour before seeding the cells. CCL21 (PeproTech, USA) and various concentrations of curcumin were added in the bottom wells of the chamber to function as the chemotactic stimuli. Human circulating fibrocytes (pretreated with different concentrations of curcumin for 72 hours) were collected and suspended in $100 \mu \mathrm{l}$ of $2 \%$ BSA in DMEM, and $1 \times 10^{6}$ viable cells were plated in the top wells of the chamber. Cells were allowed to migrate for 3 hours at $37^{\circ} \mathrm{C}$ in a $5 \% \mathrm{CO} 2$ incubator. After migration, the cells on the upper surface of the filter were wiped and the cells sticking to the lower surface were collected and counted using an Automated Cell Counter (Bio-Rad, USA). The chemotaxis 
rate was calculated by comparing the number of recruited cells with the total number of cells added into the top wells.

\section{Statistical analysis}

Results are expressed as the mean \pm S.E.M. The statistical significance of differences among three or more variables was evaluated using analysis of variance (ANOVA) by Graph-pad Prism software (version 6.1). $P$ values $<0.05$ were considered statistically significant.

\section{Results}

Identification of human circulating fibrocytes

The classical markers for human circulating fibrocytes are CD34, CD45, COL I and CCR7 [23]. Immunocytochemistry results showed that COL I , CD45 and CCR7 strongly coexpressed in human circulating fibrocytes, whereas CD34 expression was very weak (Fig. 1A). Flow cytometry results indicated that approximately $99.8 \%$ of the human circulating fibrocytes were COL I -positive, $77.8 \%$ were CD45-positive, and only $1.0 \%$ were CD34positive, suggesting that human circulating fibrocytes were successfully isolated and purified in the current study (Fig. 1B).

The effect of curcumin on the viability of human circulating fibrocytes

Our results showed that curcumin at higher concentrations (10 $\mu \mathrm{M}$ and $20 \mu \mathrm{M})$ exerted inhibitory effects on the proliferation of human circulating fibrocytes. Furthermore, this inhibitory effect was associated with an increase in curcumin concentration. Interestingly, curcumin at low concentrations $(1 \mu \mathrm{M}$ and $3 \mu \mathrm{M}$ at 24 hours or 48 hours $)$ has the potential to improve cell viability (Fig 1C). However we did not see significant differences between the treatment and control groups.

Curcumin elevates CD34 expression in human circulating fibrocytes

The expression of CD34 proved that human circulating fibrocytes were derived from bone marrow [5] and the expression of CD45 confirmed their hematopoietic origin [24]. Flow cytometry results showed that the ratio of $\mathrm{CD} 34^{+}$or $\mathrm{CD} 45^{+}$cells in the curcumin treatment group exhibited a slight increase compared to that in the control group. Specifically, CD34 expression showed a marked response to treatment with curcumin at the higher doses of $10 \mu \mathrm{M}(p<0.05)$ and $20 \mu \mathrm{M}(p<0.01)$ (Fig $2 \mathrm{~A})$, whereas CD45 did not show significant differences among these groups (Fig. 2B).

Curcumin blocks the expression of COL I and $\alpha$-SMA in human circulating fibrocytes

We next evaluated the effect of curcumin on the transdifferentiation of human circulating fibrocytes. COLI is present in scar tissue [25], which is main symptom of fibrosis development. Fibrocytes can be activated into fibroblast, which are main producer of collagen, especially COL. Our study has revealed that COLI is strongly expressed in human circulating fibrocytes, suggesting that human circulating fibrocytes could transdifferentiate into fibroblasts and induce a fibrogenic response. Furthermore, we observed a curcumin concentration-dependent reduction in COL I levels at 72 hours (Fig. 2 C)

Previous studies have shown that bone-marrow-derived circulating fibrocytes can differentiate into $\alpha-\mathrm{SMA}^{+}$myofibroblasts [26, 27], and make crucial contributions to liver fibrosis [27]. We observed that $\alpha$-SMA was strongly expressed on human circulating fibrocytes cultured for 12 days, and that there was a dose-dependent decrease in $\alpha$-SMA levels after 72-hour treatment with curcumin. Compared with the control group (94.8\%), curcumin significantly reduced the numbers of $\alpha-\mathrm{SMA}^{+}$human circulating fibrocytes at concentrations of 10 and $20 \mu \mathrm{M}$ ( $p<0.01$; Fig. 2D). 


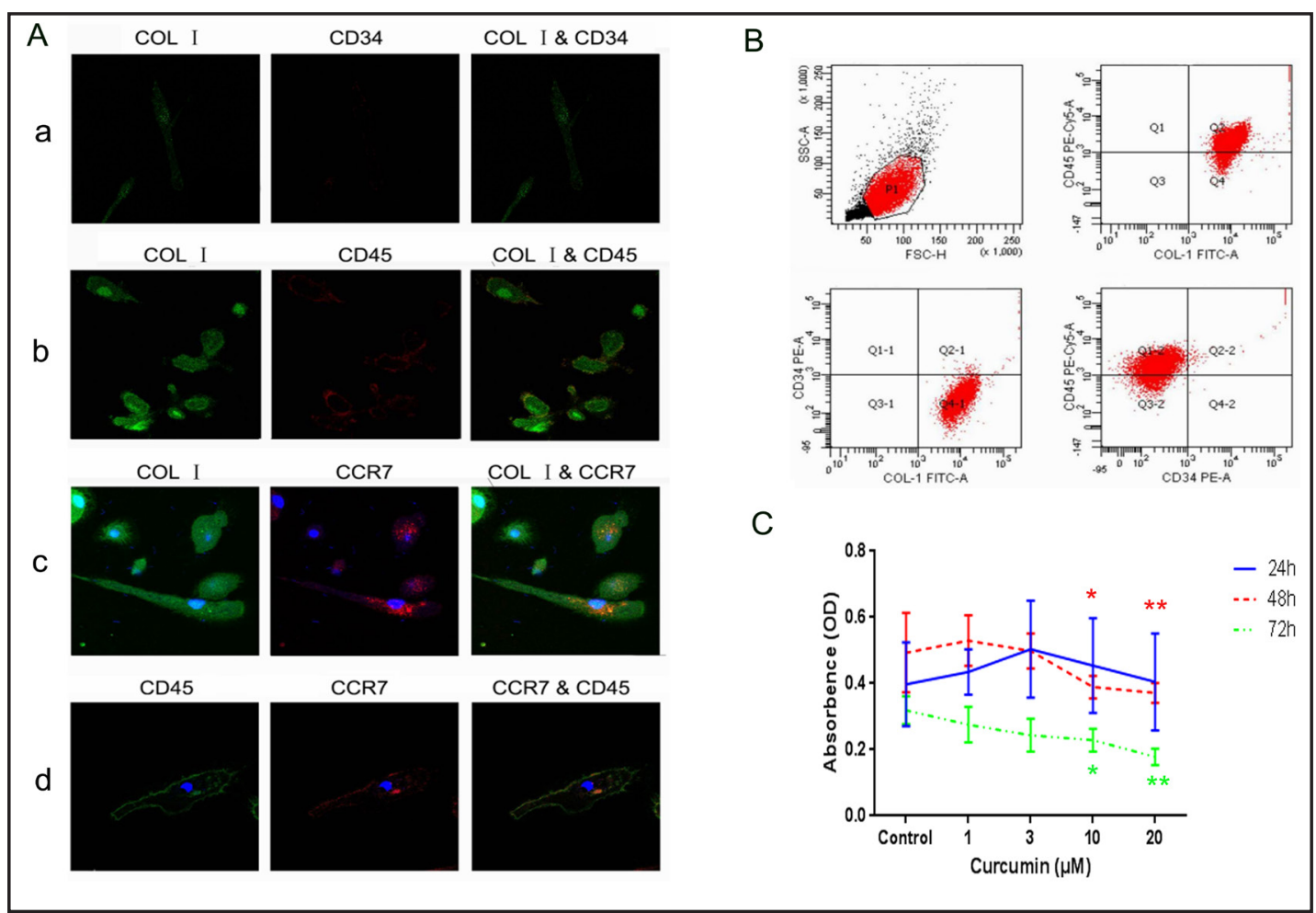

Fig. 1. Expression levels of CD34, CD45, CCR7 and COL I in human circulating fibrocytes and the effect of curcumin on the viability of human circulating fibrocytes. (A) Representative confocal images showed CD34, CD45, CCR7 and COL I coexpression in human circulating fibrocytes. Cells were costained for (a) COL I and CD34; (b) COL I and CD45; (c) COL I and CCR7; (d) CD45 and CCR7. Nuclei were stained with 4',6-diamidino-2-phenylindole (DAPI; blue). Cells were examined at 60× magnification. Results shown are representative of triple determinations ( $\mathrm{n}=3$ ). (B) Flow cytometry results show CD34, CD45 and COL I coexpression in human circulating fibrocytes. Results shown are representative of triple determinations $(\mathrm{n}=3)$. (C) CCK-8 results show that curcumin regulates the viability of human circulating fibrocytes. Data are representative of three separate experiments. ${ }^{*} p<0.05$ and ${ }^{* *} p<0.01$ indicate significant differences between control and curcumin treatment.

Curcumin suppresses the secretion of TGF- $\beta 1$ in human circulating fibrocytes

As the acknowledged fibrogenic and growth-regulating cytokine, TGF- $\beta 1$ was able to regulate multiple signaling pathways involved in fibrogenesis [28]. ELISA results showed a concentration-dependent decrease in TGF- $\beta$ l levels in cell culture media after 72-hour treatment with curcumin. This effect was not significant at low concentration of curcumin, but became prominent at concentrations ranging from 3 to $20 \mu \mathrm{M}(p<0.01$; Fig 3$)$.

Curcumin blocks the migration of human circulating fibrocytes via the CCR7/CCR21 pathway

Expressed in various lymphoid tissues and activates $\mathrm{B}$ and $\mathrm{T}$ lymphocytes, the chemokine receptor CCR7 and its ligand CCL21 play a major role in immune responses [29]. CCL21/CCR7 signaling has been demonstrated to be the primary pathway inducing the recruitment of human circulating fibrocytes into the kidney, and is thereby involved in renal fibrogenesis $[8,30]$. Given that CCR7 was found to be strongly expressed in human circulating fibrocytes in our previous study, we investigated the effect of curcumin on CCR7 levels by flow cytometry. The results showed that curcumin inhibited CCR7 expression in human circulating fibrocytes. This inhibitory effect of curcumin was initiated at doses of $1 \mu \mathrm{M}$, and was marked at doses of $3-20 \mu \mathrm{M}(p<0.01$; Fig $4 \mathrm{~A}$ and $4 \mathrm{~B})$. 


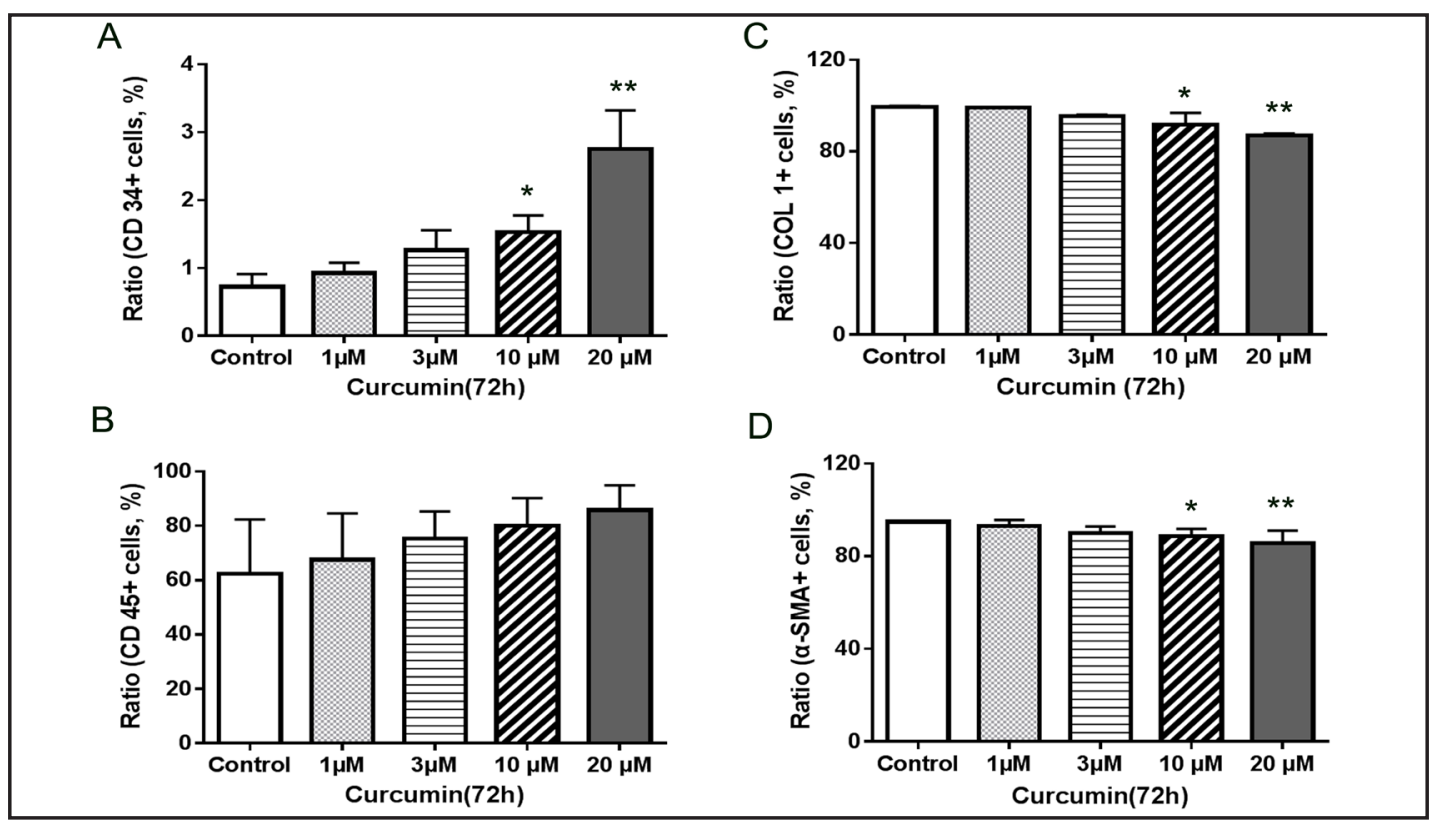

Fig. 2. Effects of curcumin on differentiation of the expression of CD34, CD45, COL I and $\alpha$-SMA expression in human circulating fibrocytes. (A-B) Flow cytometry results show that curcumin up-regulates CD34 and CD45 expression in human circulating fibrocytes. Human circulating fibrocytes were treated with curcumin $(1,3,10$ and $20 \mu \mathrm{M} ; 72$ hours). The bar diagram represents data obtained from three separate experiments, with the bars representing mean \pm SEM. (A) CD34 levels in human circulating fibrocytes stained with PE. Cells were cultured for 4 days before treatment with curcumin. (B) CD45 levels in human circulating fibrocytes stained with PE-Cy5. Cells were cultured for 6 days before treating with curcumin. (C-D) Representive flow cytometry results showed that curcumin down-regulates COL I expression and $\alpha$-SMA expression in human circulating fibrocytes. (C)Human circulating fibrocytes were treated with curcumin (1, 3, 10 and $20 \mu \mathrm{M} ; 72$ hours) and stained with FITC. Cells were cultured for 9 days before treating with curcumin. (D) Human circulating fibrocytes were treated with curcumin $(1,3,10$ and $20 \mu \mathrm{M} ; 72$ hours $)$ and stained with APC. Cells were cultured for 12 days before treatment with curcumin. ${ }^{*} p<0.05$ and ${ }^{* *} p<0.01$ indicate significant differences between control and curcumin treatment.

Fig. 3. Curcumin down-regulates TGF- $\beta 1$ secretion from human circulating fibrocytes by ELISA. Data are expressed as the mean \pm SEM and representative of three separate experiments. ${ }^{* *} p<0.01$ indicate significant differences between control and curcumin treatment.

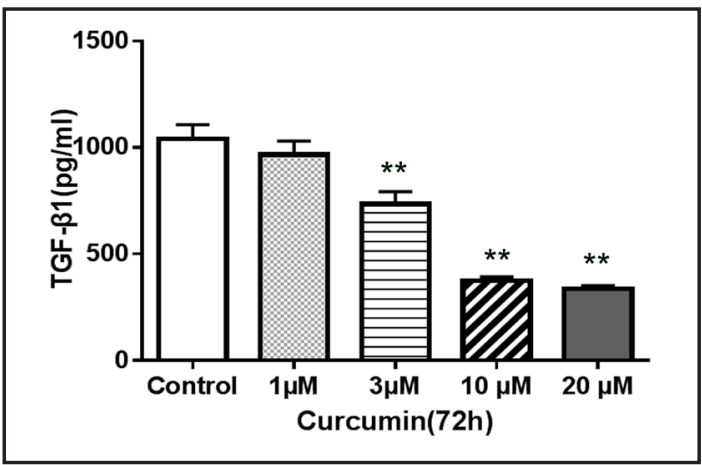

Next, we evaluated the effect of curcumin on the migration of circulating fibrocytes in response to CCL21. As shown in Figure 4C, CCL21 induced the migration of human circulating fibrocytes in a dose-dependent manner. Compared with the control group, cells migration was overactivated in response to CCL21 treatment $(1-200 \mathrm{ng} / \mathrm{ml} ; \quad p<0.05$ or $p<0.01)$. However, curcumin did not induce the migration of human circulating fibrocytes in the absence of CCL21.

Given that curcumin blocks CCR7 expression in human circulating fibrocytes, we further assessed the effect of curcumin on cell migration via the CCL21/CCR7 pathway. As shown in 
A

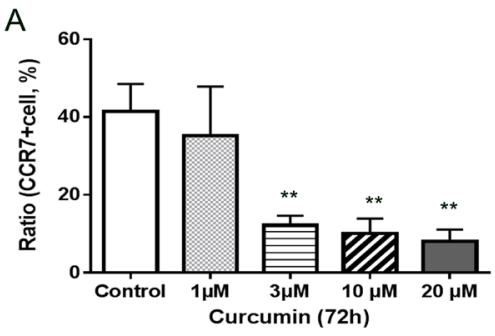

B

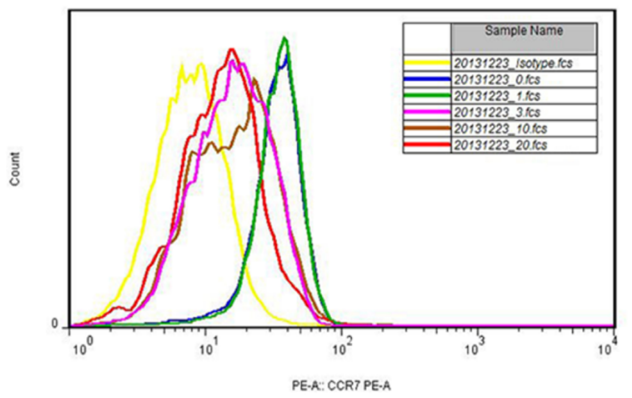

$\mathrm{C}$

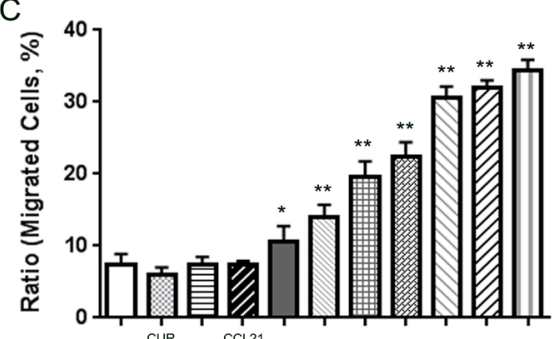

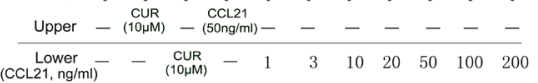

$\mathrm{D}$

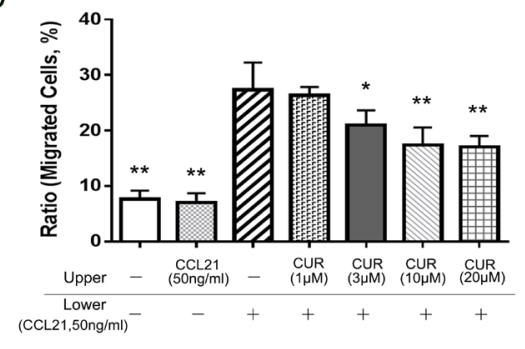

Fig. 4. Effects of curcumin on CCR7 expression and the migration of human circulating fibrocytes. (A) Curcumin down-regulates CCR7 levels in human circulating fibrocytes, as evidenced by flow cytometry. Human circulating fibrocytes were treated with curcumin $(1,3,10$ and $20 \mu \mathrm{M} ; 72$ hours) and stained with PE. Cells were cultured for 6 days before treating with curcumin. The bar diagram represents data obtained from three separate experiments, with the bars representing mean \pm SEM. (B) Representative histogram plot from three different flow cytometry assays. (C) CCL21 up-regulates the migration of human circulating fibrocytes cultured for 9 days. The left fourth column represents the control group. (D) Curcumin down-regulates the migration of human circulating fibrocytes. Cells were cultured for 6 days before treating with curcumin. Cells were pretreated with various concentrations of curcumin for 72 hours in the upper wells. Data are from three separate experiments. The left third column represents the control group. ${ }^{*} p<0.05$ and ${ }^{* *} p<0.01$ indicate significant differences between the curcumin treatment and control groups. ("CUR" means "curcumin").

Figure 4D, compared with the control group, curcumin pretreatment for 72 hours at doses of $3-20 \mu \mathrm{M}$ led to a remarkable suppression of cell migration $(3 \mu \mathrm{M}: p<0.05 ; 10-20 \mu \mathrm{M}: p<0.01)$. In addition, there was a non-significant difference between curcumin and CCL21 levels in the bottom chambers.

\section{Discussion}

In the last two decades, since the discovery of circulating fibrocytes in 1994[5], our knowledge of the pathogenesis of fibrosis has expanded dramatically. Existing in the peripheral circulation, circulating fibrocytes can be recruited into multiple organs in response to chemokines, and actively contribute to fibrogenesis. It has been proved that circulating fibrocytes play a key role in pulmonary $[6,31,32]$, renal $[7,8]$, skin $[7,33]$, hepatic $[7,9]$ and cardiac $[7,11]$ fibrosis. Consequently, it can be speculated that circulating fibrocytes could be one of the common targets for anti-fibrotic therapy in various organs.

As bone marrow-derived mesenchymal progenitors, circulating fibrocytes are a special population of peripheral leukocyte subsets with hallmarks of fibrotic pathologies, co-expressing hematopoietic stem cell antigens and markers of the monocyte lineage as well as fibroblasts[2]. Fibrocytes are markedly positive for specific molecular markers, including leukocyte (CD45), monocyte (CD11a, CD11b, CD13), and bone marrow stem cell 
markers (CD34), chemokine receptors (CXCR4, CCR7, CCR2, CXCR6), major histocompability complex (MHC I , MHC II ), and mesenchymal cell makers (collagen I, pro-collagen I, collagen III, vimentin, prolyl4-hydroxylase and $\alpha$-SMA) $[2,12,34]$. The combination of collagen production and hematogenous origin features as a good standard to identify circulating fibrocytes in most settings, i.e. co-expression of CD34 or CD45 and COL I or pro-COL I[2].

CD34 belongs to a special class of transmembrane glycoproteins that are heavily glycosylated, and has the capacity to transfer hematopoietic cells from bone marrow into peripheral blood [35]. In our study, the expression of CD34 indicated the hematogenous stem origin of human circulating fibrocytes, consistent with previous studies[36]. We found that only $1.0 \%$ of the cells were CD34+ in the early period (less than $2.99 \%$ in a study by Liu et al [36]). It has been reported that the proportion of $\mathrm{CD} 34^{+}$cells in peripheral blood ranges from $0.01 \%$ to $0.1 \%$ by flow cytometry [37]. We believe that the level of CD34 ${ }^{+}$cells may vary due to differences in blood separation methods. The expression of CD45 indicates the panhematogenous origin of human circulating fibrocytes. The high level of COLI demonstrated that human circulating fibrocytes could transdifferentiate into fibroblasts, and thereby play a role in fibrogenesis. Flow cytometry results showed that $77.8 \%$ of cells were both CD $45^{-}$ and $\mathrm{COLI}^{+}$, typical of human circulating fibrocytes.

In this study, we have discovered that curcumin treatment (72 hours, $20 \mu \mathrm{M}$ ) significantly down-regulated COLI, $\alpha$-SMA, and CCR7 levels, and blocked TGF- $\beta 1$ secretion, as well as the migration of human circulating fibrocytes. Curcumin remarkably improved the expression of CD34 on human circulating fibrocytes, but did not significantly increase CD45 levels.

CD34 and CD45 are hematopoietic markers, COLI is the hallmark of fibroblasts, and $\alpha$-SMA is the classical marker of myofibroblasts. The modulation of curcumin on these surface phenotypes indicated that curcumin was able to maintain the hematopoietic features of cells and suppress the transdifferentiation of human circulating fibrocytes into fibroblasts or myofibroblasts.

TGF- $\beta 1$ can regulate the synthesis and degradation of ECM and induce the activity of other fibrogenic cytokines that contribute to the development of fibrosis [28]. It has been reported that TGF- $\beta 1$ can enhance the expression of COLI and $\alpha$-SMA, and induce the differentiation of $\mathrm{CD} 45^{+} \mathrm{CD} 34^{+} \mathrm{COLI}^{+}$fibrocytes to myofibroblasts by the activation of the Smad $2 / 3$ and SAPK/ JNK signaling pathways [26]. In the present study, curcumin exhibited anti-fibrotic effects by inhibiting TGF- $\beta$ l secretion in human circulating fibrocytes. Furthermore, the effects of curcumin on the expressions of CD45, CD34, COL I and $\alpha$-SMA expressions may be mediated via regulating TGF- $\beta 1$ secretion, suggesting that curcumin could block the differentiation of human circulating fibrocytes by reducing the secretion of TGF- $\beta 1$.

Recent studies indicated that the CCL21 and CCR7 signaling pathways induce the trafficking of circulating fibrocytes in pulmonary [38] and renal fibrogenesis [39]. In a murine UUO model, a large number of circulating CD45+/COLI+ fibrocytes were shown to be able to infiltrate the renal interstitium via CCL21-positive HEV-like vessels [8]. Most fibrocytes were positive for CCR7, and the inhibition of CCL21/CCR7 signaling reduced the number of infiltrating fibrocytes, and blocked the pathogenesis of renal fibrosis in UUO-treated mice [19]. Here, flow cytometry results showed a dose-dependent inhibitory effect of curcumin on CCR7 expression, and revealed that pretreatment with curcumin for 72 hours effectively inhibited the migration of human circulating fibrocytes in response to CCL21. Taken together, these results suggested that curcumin may block the differentiation and migration of human circulating fibrocytes by down-regulating the level of CCR7 via the CCL21/CCR7 pathways.

In conclusion, our study revealed that curcumin can reduce the migration, and downregulate the COL I, $\alpha$-SMA and CCR7 levels, as well as the TGF- $\beta$ I secretion in human circulating fibrocytes. The possible mechanism mediating these effects may be through regulating CCR7/CCL21 signaling pathway, mainly via reducing CCR7 expression. These observed effects of curcumin may contribute to resolve fibrosis via suppressing TGF- $\beta 1$ secretion. This also suggest that curcumin may exert its anti-fibrotic effects in various organs by targeting circulating fibrocytes. 
Fu et al.: Curcumin and Human Circulating Fibrocytes

\section{Disclosure Statement}

All authors declare that they have no any actual or potential conflict of interest including any financial, personal or other relationships with other people or organizations within three years of beginning the submitted work that could inappropriately influence, or be perceived to influence, their work.

\section{Acknowledgements}

This study was supported by the National Natural Science Foundation of China (No. 81173642, No. 81273995, No. 81274041) and by the 111 Project (No. B07007).

\section{References}

1 Kendall RT, Feghali-Bostwick CA: Fibroblasts in fibrosis: Novel roles and mediators. Front Pharmacol 2014;5:123.

- Herzog EL, Bucala R: Fibrocytes in health and disease. Exp Hematol 2010;38:548-556.

3 http://en.wikipedia.org/wiki/Fibrosis:

4 Pottier N, Cauffiez C, Perrais M, Barbry P, Mari B: Fibromirs: Translating molecular discoveries into new anti-fibrotic drugs. Trends Pharmacol Sci 2014;35:119-126.

5 Bucala R, Spiegel LA, Chesney J, Hogan M, Cerami A: Circulating fibrocytes define a new leukocyte subpopulation that mediates tissue repair. Mol Med 1994;1:71-81.

6 Fujiwara A, Kobayashi H, Masuya M, Maruyama M, Nakamura S, Ibata H, Fujimoto H, Ohnishi M, Urawa M, Naito M, Takagi T, Kobayashi T, Gabazza EC, Takei Y, Taguchi O: Correlation between circulating fibrocytes, and activity and progression of interstitial lung diseases. Respirology 2012;17:693-698.

7 Wagner B, Tan C, Barnes JL, Ahuja S, Davis TL, Gorin Y, Jimenez F: Nephrogenic systemic fibrosis: Evidence for oxidative stress and bone marrow-derived fibrocytes in skin, liver, and heart lesions using a 5/6 nephrectomy rodent model. Am J Pathol 2012;181:1941-1952.

8 Sakai N, Wada T, Yokoyama H, Lipp M, Ueha S, Matsushima K, Kaneko S: Secondary lymphoid tissue chemokine (slc/ccl21)/ccr7 signaling regulates fibrocytes in renal fibrosis. Proc Natl Acad Sci U S A 2006;103:14098-14103.

9 Kallis YN, Scotton CJ, Mackinnon AC, Goldin RD, Wright NA, Iredale JP, Chambers RC, Forbes SJ: Proteinase activated receptor 1 mediated fibrosis in a mouse model of liver injury: A role for bone marrow derived macrophages. PLoS One 2014;9:e86241.

10 Jarcuska P, Janicko M, Veseliny E, Jarcuska P, Skladany L: Circulating markers of liver fibrosis progression. Clin Chim Acta 2010;411:1009-1017.

11 Williams SM, Golden-Mason L, Ferguson BS, Schuetze KB, Cavasin MA, Demos-Davies K, Yeager ME, Stenmark KR, McKinsey TA: Class i hdacs regulate angiotensin ii-dependent cardiac fibrosis via fibroblasts and circulating fibrocytes. J Mol Cell Cardiol 2014;67:112-125.

12 Quan TE, Cowper S, Wu SP, Bockenstedt LK, Bucala R: Circulating fibrocytes: Collagen-secreting cells of the peripheral blood. Int J Biochem Cell Biol 2004;36:598-606.

13 Grieb G, Steffens G, Pallua N, Bernhagen J, Bucala R: Circulating fibrocytes--biology and mechanisms in wound healing and scar formation. Int Rev Cell Mol Biol 2011;291:1-19.

14 Zhang DW, Fu M, Gao SH, Liu JL: Curcumin and diabetes: A systematic review. Evid Based Complement Alternat Med 2013;2013:636053.

15 Meng Z, Yu XH, Chen J, Li L, Li S: Curcumin attenuates cardiac fibrosis in spontaneously hypertensive rats through ppar-gamma activation. Acta Pharmacol Sin 2014;35:1247-1256.

16 Yao QY, Xu BL, Wang JY, Liu HC, Zhang SC, Tu CT: Inhibition by curcumin of multiple sites of the transforming growth factor-beta1 signalling pathway ameliorates the progression of liver fibrosis induced by carbon tetrachloride in rats. BMC Complement Altern Med 2012;12:156. 
17 Zhang Z, Guo Y, Zhang S, Zhang Y, Wang Y, Ni W, Kong D, Chen W, Zheng S: Curcumin modulates cannabinoid receptors in liver fibrosis in vivo and inhibits extracellular matrix expression in hepatic stellate cells by suppressing cannabinoid receptor type-1 in vitro. Eur J Pharmacol 2013;721:133-140.

18 Yao Q, Lin Y, Li X, Shen X, Wang J, Tu C: Curcumin ameliorates intrahepatic angiogenesis and capillarization of the sinusoids in carbon tetrachloride-induced rat liver fibrosis. Toxicol Lett 2013;222:72-82.

19 Kuwabara N, Tamada S, Iwai T, Teramoto K, Kaneda N, Yukimura T, Nakatani T, Miura K: Attenuation of renal fibrosis by curcumin in rat obstructive nephropathy. Urology 2006;67:440-446.

20 Soetikno V, Sari FR, Lakshmanan AP, Arumugam S, Harima M, Suzuki K, Kawachi H, Watanabe K: Curcumin alleviates oxidative stress, inflammation, and renal fibrosis in remnant kidney through the nrf2-keap1 pathway. Mol Nutr Food Res 2013;57:1649-1659.

-21 Avasarala S, Zhang F, Liu G, Wang R, London SD, London L: Curcumin modulates the inflammatory response and inhibits subsequent fibrosis in a mouse model of viral-induced acute respiratory distress syndrome. PLoS One 2013;8:e57285.

22 Zhang D, Huang C, Yang C, Liu RJ, Wang J, Niu J, Bromme D: Antifibrotic effects of curcumin are associated with overexpression of cathepsins $\mathrm{k}$ and $\mathrm{l}$ in bleomycin treated mice and human fibroblasts. Respir Res 2011;12:154.

23 Strieter RM, Keeley EC, Burdick MD, Mehrad B: The role of circulating mesenchymal progenitor cells, fibrocytes, in promoting pulmonary fibrosis. Trans Am Clin Climatol Assoc 2009;120:49-59.

24 Peng H, Herzog EL: Fibrocytes: Emerging effector cells in chronic inflammation. Curr Opin Pharmacol 2012;12:491-496.

25 Mescher AL: Junqueira's basic histology. McGraw-Hill Medical;, 2013.

26 Hong KM, Belperio JA, Keane MP, Burdick MD, Strieter RM: Differentiation of human circulating fibrocytes as mediated by transforming growth factor-beta and peroxisome proliferator-activated receptor gamma. J Biol Chem 2007;282:22910-22920.

27 Forbes SJ, Russo FP, Rey V, Burra P, Rugge M, Wright NA, Alison MR: A significant proportion of myofibroblasts are of bone marrow origin in human liver fibrosis. Gastroenterology 2004;126:955-963.

28 Samarakoon R, Overstreet JM, Higgins PJ: Tgf-beta signaling in tissue fibrosis: Redox controls, target genes and therapeutic opportunities. Cell Signal 2013;25:264-268.

29 Comerford I, Harata-Lee Y, Bunting MD, Gregor C, Kara EE, McColl SR: A myriad of functions and complex regulation of the ccr7/ccl19/ccl21 chemokine axis in the adaptive immune system. Cytokine Growth Factor Rev 2013;24:269-283.

30 Abe R, Donnelly SC, Peng T, Bucala R, Metz CN: Peripheral blood fibrocytes: Differentiation pathway and migration to wound sites. J Immunol 2001;166:7556-7562.

-31 Saunders R, Siddiqui S, Kaur D, Doe C, Sutcliffe A, Hollins F, Bradding P, Wardlaw A, Brightling CE: Fibrocyte localization to the airway smooth muscle is a feature of asthma. J Allergy Clin Immunol 2009;123:376-384.

-32 Strieter RM, Keeley EC, Hughes MA, Burdick MD, Mehrad B: The role of circulating mesenchymal progenitor cells (fibrocytes) in the pathogenesis of pulmonary fibrosis. J Leukoc Biol 2009;86:1111-1118.

-33 Oh MH, Oh SY, Yu J, Myers AC, Leonard WJ, Liu YJ, Zhu Z, Zheng T: Il-13 induces skin fibrosis in atopic dermatitis by thymic stromal lymphopoietin. J Immunol 2011;186:7232-7242.

-34 Andersson-Sjoland A, Nihlberg K, Eriksson L, Bjermer L, Westergren-Thorsson G: Fibrocytes and the tissue niche in lung repair. Respir Res 2011;12:76.

35 Yin T, Li L: The stem cell niches in bone. J Clin Invest 2006;116:1195-1201.

-36 Peng Liu GH, Jie Feng, Yan Jin: Influence of circulating fibrocytes on the growth, proliferation and migration of keratinocytes and fibroblasts. Journal of Applied BiomedicineJournal of Applied Biomedicine 2012;10:917.

37 Barnett D, Janossy G, Lubenko A, Matutes E, Newland A, Reilly JT: Guideline for the flow cytometric enumeration of cd34+ haematopoietic stem cells. Prepared by the cd34+ haematopoietic stem cell working party. General haematology task force of the british committee for standards in haematology. Clin Lab Haematol 1999;21:301-308.

-38 Habiel DM, Hogaboam C: Heterogeneity in fibroblast proliferation and survival in idiopathic pulmonary fibrosis. Front Pharmacol 2014;5:2.

39 Wada T, Sakai N, Matsushima K, Kaneko S: Fibrocytes: A new insight into kidney fibrosis. Kidney Int 2007;72:269-273. 